Pacific Journal of Mathematics

THE EQUIVARIANT THOM ISOMORPHISM THEOREM 


\title{
THE EQUIVARIANT THOM ISOMORPHISM THEOREM
}

\author{
S. R. COSTENoble AND S. WANER
}

\begin{abstract}
In this paper we extend ordinary $R O(G)$-graded cohomology to a theory graded on virtual $G$-bundles over a $G$-space and show that a Thom Isomorphism theorem for general $G$-vector bundles results. Our approach uses Elmendorf's topologized spectra. We also show that the grading can be reduced from the group of virtual $G$-vector bundles over a space to a quotient group, using ideas from a new theory of equivariant orientations. As an application of the Thom Isomorphism theorem, we give a new calculation of the additive structure of the equivariant cohomology of complex projective spaces for $G=\mathrm{Z} / p$, partly duplicating and partly extending a recent calculation done by Lewis.
\end{abstract}

1. Introduction. Nonequivariantly, the Thom Isomorphism plays an important role in calculations of cohomology groups. Equivariantly, the Thom Isomorphism is a more elusive beast; $\mathbb{Z}$-graded ordinary cohomology is inadequate for its formulation, as can be seen by the fact that the cohomology of the one-point compactification of a representation is usually radically different from the cohomology of a point. Extending the $\mathbb{Z}$-graded theory of an $R O(G)$-graded theory [LMM] is still inadequate; the cohomology of the one-point compactification of a fiber of a bundle is now the suspension of that of a point, but the degree of the suspension depends on the group action on the fiber, and in general this varies as we move around in the base space.

Here we shall extend the $R O(G)$-graded theory to a theory graded on virtual $G$-bundles, and show that we can then prove a Thom Isomorphism theorem for general $G$-vector bundles. The use of virtual $G$-bundles for grading equivariant cohomology is not entirely newCrabb [C], for example, considers stable cohomotopy, for $\mathbb{Z} / 2$-actions, indexed in this way. Our approach differs in that it uses the topologized spectra that Elmendorf introduced in [E]. Although we could phrase our definitions in terms of parametrized spectra, we have chosen instead to take a more elementary approach. We also show that the grading can be reduced from virtual $G$-vector bundles to their "groupoid representations," gadgets that capture the orientability data of the bundles. This is a first step toward the construction of an ordinary theory indexed on groupoid representations; these representations are fundamental to equivariant orientation theory as presented in $[\mathbf{C M W}$. 
As an application of the equivariant Thom Isomorphism theorem, we give a new calculation of the additive structure of the equivariant cohomology of complex projective space for $G=\mathbb{Z} / p$. This partly duplicates and partly extends a recent calculation done by Lewis [L1] using different methods.

Along the way, we construct classifying spaces for arbitrary groupoids over the orbit category and prove a classification theorem (Theorem 3.8).

For the next section of this paper $G$ may be any compact Lie group, but we will specialize to finite groups in the third and later sections.

2. Cohomology theories graded on $K O_{G}(X)$. We use Elmendorf's notion of an equivariant spectrum [E], which we recall here. Let $\mathscr{U}$ be a $G$-universe, that is, a countably infinite dimensional orthogonal $G$-representation which contains infinitely many copies of each finite dimensional representation of $G$. We recall that a $G$-spectrum $E$ in the sense of Lewis, May and Steinberger [MS] consists of a collection of based $G$-spaces $E(V)$, one for each finite dimensional $G$ invariant subspace $V$ of $\mathscr{U}$, together with a collection of compatible $G$-homeomorphisms $E(V) \cong \Omega^{W}(V \oplus W)$ for $V \perp W$. (see [MS] for details.) Elmendorf [E] replaces the spaces $E(V)$ with $G$-fiber bundles $p: E_{n} \rightarrow B_{G} O_{n}$ over the Grassmannian of $n$-planes in $\mathscr{U}$, with fiber over every $G$-invariant $V$ a copy of $E(V)$. Each bundle is also equipped with a $G$-section $\sigma$ giving the basepoint in each fiber. Elmendorf shows that any $G$-spectrum in the sense of [LMS] gives rise to a unique spectrum in his sense.

We want to describe how to define the $E$-cohomology of a space $X$, graded on $K O_{G}(X)$. We will describe here how to grade on pairs of subbundles of $X \times U$; in the Appendix we will refine this, and say in exactly what sense we can grade on $K O_{G}(X)$.

Let $X$ be an unbased $G$-space and let $\xi$ and $\zeta$ be finite-dimensional $G$-subbundles of $X \times \mathscr{U}$. Then $\xi$ is classified by the Gauss map $\tilde{\xi}: X \rightarrow B_{G} O_{n}$. If $E$ is a $G$-spectrum, we define $E_{G}^{\xi-\zeta}(X)$ as the group of $G$-homotopy classes of section-preserving $G$-maps $c$ making the following diagram commute:

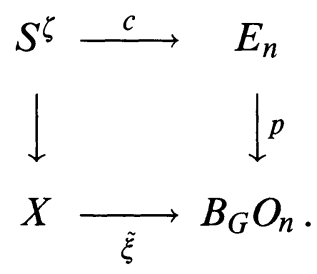


Here, $S^{\zeta}$ is the fiberwise one-point compactification of $\zeta$, and the homotopies are required to preserve sections and to cover $\tilde{\xi}$. The additive structure is a consequence of the structure of the spectrum $E$. This construction is functorial in $X$; we will give more details on this point in the Appendix. Note that we are using the notation $\xi-\zeta$ to mean simply the ordered pair $(\xi, \zeta)$.

We now generalize to pairs of $G$-spaces, and based $G$-spaces in particular. As in $[\mathbf{J}]$, a $G$-ex-space is a $G$-map $f: Y \rightarrow X$ with a given $G$-section; we also refer to this as a based-G-space over $X$ if we wish to emphasize $X$. Many of the constructions we use here involving ex-spaces can be found in [J]. Given a $G$-ex-space $Y \rightarrow X$ as well as $G$-vector bundles $\xi$ and $\zeta$ over $X$ as above, define $\widetilde{E}_{G}^{\xi-\zeta}(Y \rightarrow X)$ as the collection of $G$-homotopy classes of section-preserving $G$-maps $c$ making the following diagram commute:

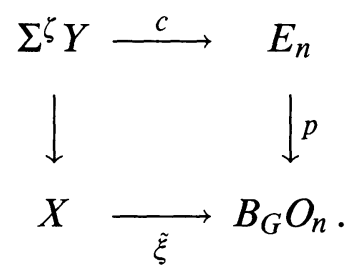

Here, $\Sigma^{\zeta} Y$ is the fiberwise smash product of $Y$ with $S^{\zeta}$ over $X$. This generalizes the special case described above: just take $Y$ to be the space $X^{+}$, which is $X$ with a disjoint section. Given a pair of $G$-spaces $(Y, B)$ over $X$, let $C(Y, B) \rightarrow X$ denote the fiberwise cone of the inclusion $B^{+} \rightarrow Y^{+}$(note that the arrow is part of the notation), and define $E_{G}^{\xi-\zeta}((Y, B) \rightarrow X)=\widetilde{E}_{G}^{\xi-\zeta}(C(Y, B) \rightarrow X)$. In particular, if $A \subset X$, define $E_{G}^{\xi-\zeta}(X, A)=\widetilde{E}_{G}^{\xi-\zeta}(C,(X, A) \rightarrow X)$ and $\widetilde{E}_{G}^{\xi-\zeta}(X)=E_{G}^{\xi-\zeta}(X, *)$ if $X$ is based at *.

We have the following form of suspension isomorphism.

Proposition 2.1. If $\eta$ is any $G$-subbundle of $X \times \mathscr{U}$ fiberwise perpendicular to both $\xi$ and $\zeta$, then

$$
\widetilde{E}_{G}^{\xi-\zeta}(Y \rightarrow X) \cong \widetilde{E}_{G}^{(\eta+\xi)-\zeta}\left(\Sigma^{\eta} Y \rightarrow X\right) .
$$

Proof. We recall from [E] the definition of the structure maps of $E$. Let $B_{G} O_{n, m}$ be the Grassmannian space of pairs of mutually orthogonal subspaces of $\mathscr{U}$ of dimension $(n, m)$. There are maps $p_{1}: B_{G} O_{n, m} \rightarrow B_{G} O_{n}$ and $s: B_{G} O_{n, m} \rightarrow B_{G} O_{n+m}$, given by projection and sum respectively. Let $\gamma_{n}$ denote the canonical bundle over $B_{G} O_{n}$, 
and also let it denote $p_{1}^{*} \gamma_{n}$; similarly let $\gamma_{m}$ denote the canonical bundle over $B_{G} O_{n, m}$ given by the second vector space. Then the structure maps of $E$ are maps $\Sigma^{\gamma} p_{1}^{*} E_{n} \rightarrow s^{*} E_{n+m}$ over $B_{G} O_{n, m}$, such that the adjoint maps $p_{1}^{*} E_{n} \rightarrow \Omega^{\gamma} S^{*} E_{n+m}$ are homeomorphisms.

Given then a map $c: \Sigma^{\zeta} Y \rightarrow E_{n}$ covering $\tilde{\xi}$, we have a map $\Sigma^{\eta} c: \Sigma^{\zeta} \Sigma^{\eta} Y \rightarrow \Sigma^{\gamma_{m}} p_{1}^{*} E_{n}$ covering the map $(\tilde{\xi}, \tilde{\eta}): X \rightarrow B_{G} O_{n, m}$ induced by the classifying maps for $\xi$ and $\eta$. The composite $\Sigma^{\zeta} \Sigma^{\eta} Y \rightarrow$ $\Sigma^{\gamma} p_{1}^{*} E_{n} \rightarrow s^{*} E_{n+m} \rightarrow E_{n+m}$ covers $\widetilde{\xi}+\tilde{\eta}: X \rightarrow B_{G} O_{n+m}$ classifying the sum of the bundles. This describes the map $\widetilde{E}_{G}^{\xi-\zeta}(Y \rightarrow X) \rightarrow$ $\widetilde{E}_{G}^{(\eta+\xi)-\zeta}\left(\Sigma^{\eta} Y \rightarrow X\right)$. That this is an isomorphism can now be checked from the fact that $p_{1}^{*} E_{n} \rightarrow \Omega^{\gamma_{m} S^{*} E_{n+m}}$ is a homeomorphism.

It now follows from our definition of the cohomology of pairs, and from $[\mathbf{J}, 7.2]$, that we have the usual long extact sequence associated to a pair.

In order to reconcile Proposition 2.1 with the usual suspension isomorphism, involving the suspension of $X$, we need the following excision result. Let $U$ be an open $G$-invariant subspace of $X$ and let $(Y, B)$ be a pair of spaces over $X$. We say that $\left(Y, B ; p^{-1}(U)\right)$ is an excisive triad over $X$ if $\bar{U}$ has a neighborhood $W$ in $X$ such that $p^{-1}(W) \subset \operatorname{Int} B$.

LEMMA 2.2 (Excision). Let $\left(Y, B ; p^{-1}(U)=V\right)$ be an excisive triad over $X$. If $\alpha=\xi-\zeta$ is a difference of $G$-bundles over $X$, then

$$
\widetilde{E}_{G}^{\alpha}(C(Y, B) \rightarrow X) \cong \widetilde{E}_{G}^{\alpha}(C(Y-V, B-V) \rightarrow X-U) .
$$

In particular, if $(X, A ; U)$ is an excisive triad, then

$$
E_{G}^{\alpha}(X, A) \cong E_{G}^{\alpha}(X-U, A-U) .
$$

(Here, we are using $\alpha$ to denote both $\xi-\zeta$ and its restriction to $X-U$.

Proof. If $f: \Sigma^{\zeta} C(Y, B) \rightarrow E_{n}$, the hypothesis allows us to see that $f$ is fiberwise homotopic to a map that takes $\Sigma^{\zeta} q^{-1}(\bar{U})$ to the fiberwise basepoints, where $q: \Sigma^{\zeta} C(Y, B) \rightarrow X$ is the projection. The result follows from this.

Note that the lemma allows one to extend the grading in $E_{G}^{*}(X, A)$ from $G$-bundles over $X$ to $G$-bundles over $X-U$. This is needed in formulating our first version of the Thom Isomorphism theorem, 
which generalizes the nonequivariant version with twisted coefficients, as well as the usual suspension isomorphism.

Proposition 2.3 (Thom Isomorphism-Twisted Form). If $\eta$ is any $G$-subbundle of $X \times \mathscr{U}$ fiberwise perpendicular to both $\xi$ and $\zeta$, then there is a natural isomorphism

$$
E_{G}^{\xi-\zeta}(X) \cong \widetilde{E}_{G}^{(\eta+\xi)-\zeta}(T \eta)
$$

where $T \eta$ is the Thom space of $\eta$. (See the comment at the end of the proof for the definition of the second group.)

Proof. The result is a consequence of the following chain of natural isomorphisms in which $D(\eta)$ and $S(\eta)$ represent the unit disc and sphere bundles of $\eta$ respectively, and $\alpha=\xi-\zeta$.

$$
\begin{aligned}
E_{G}^{\alpha}(X) & \cong \widetilde{E}_{G}^{\alpha+\eta}\left(\Sigma^{\eta} X^{+} \rightarrow X\right) \\
& \cong \widetilde{E}_{G}^{\alpha+\eta}(C(D(\eta), S(\eta)) \rightarrow X) \\
& \cong \widetilde{E}_{G}^{\alpha+\eta}(C(D(\eta), S(\eta)) \rightarrow D(\eta)) \\
& =E_{G}^{\alpha+\eta}(D(\eta), S(\eta)) \\
& \cong \widetilde{E}_{G}^{\alpha+\eta}(T \eta)
\end{aligned}
$$

The third isomorphism follows from the $G$-ex-homotopy equivalence of $C(D(\eta), S(\eta)) \rightarrow X$ and $C(D(\eta), S(\eta)) \rightarrow D(\eta)$ and Theorem A.3(iii) in the Appendix. The equality on the fourth line is by definition of the notation. Note that the last step is essentially excision and $G$-ex-homotopy invariance if $\alpha+\eta$ extends to a bundle over the Thom space. More precisely, let $U=T \eta-D_{2} \eta$, where $D_{2}$ denotes the disc bundle of radius 2 . Then $(T \eta, \overline{T \eta-D \eta} ; U)$ is excisive in the sense of Lemma 2.2. Hence, by Lemma 2.2 and Theorem A.3(ii), we have

$$
\begin{aligned}
\widetilde{E}_{G}^{\alpha+\eta}(T \eta) & \cong E_{G}^{\alpha+\eta}(T \eta, \overline{T \eta-D \eta}) \\
& \cong E_{G}^{\alpha+\eta}(T \eta-U, \overline{T \eta-D \eta}-U) \\
& \cong E_{G}^{\alpha+\eta}(D(\eta), S(\eta))
\end{aligned}
$$

If $\alpha+\eta$ fails to extend, we can define $\widetilde{E}_{G}^{\alpha+\eta}(T \eta)$ to be

$$
\widetilde{E}_{G}^{\alpha+\eta}(C(D(\eta), S(\eta)),
$$

following the remark after Lemma 2.2. 
The next result permits us to regard the theory as being graded on $K O_{G}(X)$ rather than on formal differences of representations.

Proposition 2.4. Let $(\xi, \zeta)$ and $\left(\xi^{\prime}, \zeta^{\prime}\right)$ be pairs of representations with $[\xi-\zeta]=\left[\xi^{\prime}-\zeta^{\prime}\right]$ in $K O_{G}(X)$. Then $\widetilde{E}_{G}^{\xi-\zeta}(f) \cong \widetilde{E}_{G}^{\xi^{\prime}-\zeta^{\prime}}(f)$ for any $G$-space $f: Y \rightarrow X$ over $X$.

Proof. Since $[\xi-\zeta]=\left[\xi^{\prime}-\zeta^{\prime}\right]$ in $K O_{G}(X)$, there is a $G$-bundle $\eta$ with $\xi \oplus \zeta^{\prime} \oplus \eta \cong \xi^{\prime} \oplus \zeta \oplus \eta$. One has

$$
\begin{aligned}
\widetilde{E}_{G}^{\xi-\zeta}(Y \rightarrow X) & \cong \widetilde{E}_{G}^{\xi}\left(\Sigma^{\zeta} Y \rightarrow X\right) \\
& \cong \widetilde{E}_{G}^{\xi \oplus \zeta^{\prime} \oplus \eta}\left(\Sigma^{\zeta \oplus \zeta^{\prime} \oplus \eta} Y \rightarrow X\right) \\
& \cong \widetilde{E}_{G}^{\xi^{\prime} \oplus \zeta \oplus \eta}\left(\Sigma^{\zeta \oplus \zeta^{\prime} \oplus \eta} Y \rightarrow X\right) \\
& \cong \widetilde{E}_{G}^{\xi^{\prime}-\zeta^{\prime}}(Y \rightarrow X) .
\end{aligned}
$$

Although we have not specified choices of isomorphisms in the proof above, it is possible to make $\widetilde{E}_{G}^{*}(-)$ into a functor on the category of pairs $(f, \alpha)$ where $f$ is a $G$-space over $X$ ( $X$ allowed to vary) and where $\alpha$ is a "virtual bundle" over $X$. This is deliberately vague, but the Appendix gives the details. It does not appear that we can create a well-defined functor on the category of pairs $(f, \alpha)$, where $\alpha \in K O_{G}(X)$; some bundle maps must be specified. However, we will write $\widetilde{E}_{G}^{\alpha}(Y \rightarrow X)$ when $\alpha \in K O_{G}(X)$, with the understanding that this is natural in $f$ and $\alpha$ only when we specify a particular difference of subbundles of $X \times \mathscr{U}$ representing $\alpha$ and particular pairs of bundle maps.

3. Classifying spaces and ordinary cohomology graded on $K O_{G}(X)$. We shall see in this section that, when the theory is ordinary cohomology, the grading introduced in the previous section reduces to a quotient of $K O_{G}(X)$, and that this gives a Thom Isomorphism where the shift in the grading is by the "dimension" of the bundle, rather than the bundle itself. We restrict attention here to $G$ finite, the details in the compact Lie case being somewhat messy. Let $H T$ be the $G$-spectrum representing ordinary $R O(G)$-graded equivariant cohomology with coefficients in the Mackey functor $T$. (See, for example, [LMM] or $[\mathbf{C W}]$.)

In order to describe the reduction of the grading, we first recall the following definitions from [CMW], which are fundamental to the theory of equivariant orientations presented there. If $X$ is a 
$G$-space, the fundamental groupoid $\pi(X ; G)$ of $X$ is the category whose objects are the $G$-maps $x: G / H \rightarrow X$, where $H$ ranges over the subgroups of $G$; equivalently, $x$ is a point in $X^{H}$. A morphism $x \rightarrow y, y: G / K \rightarrow X$, is the equivalence class of a pair $(\sigma, \omega)$, where $\sigma: G / H \rightarrow G / K$ is a $G$-map, and where $\omega: G / H \times I \rightarrow X$ is a $G$-homotopy from $x$ to $y \circ \sigma$. Two such maps are equivalent if there is a $G$-homotopy $k: \omega \simeq \omega^{\prime}$ such that $k(\alpha, 0, t)=x(\alpha)$ and $k(\alpha, 1, t)=y \circ \sigma(\alpha)$ for $\alpha \in G / H$ and $t \in I$.

Let $\mathscr{G}$ be the category of $G$-orbits and $G$-maps between them. There is a functor $\phi: \pi(X ; G) \rightarrow \mathscr{G}$, given by $\phi(x: G / H \rightarrow X)=$ $G / H$ on objects, and by $\phi(\sigma, \omega)=\sigma$ on morphisms. This turns $\pi(X ; G)$ into a groupoid over $\mathscr{G}$ in the sense of [CMW]. Precisely:

Definition 3.1. A groupoid over a small category $\mathscr{B}$ consists of a small category $\mathscr{C}$ and a functor $\phi: \mathscr{C} \rightarrow \mathscr{B}$ satisfying the following properties:

(a) For each object $b$ of $\mathscr{B}$, the fiber $\phi^{-1}\left(\mathrm{id}_{b}\right)$ is a groupoid in the classical sense (i.e., all morphisms in $C$ covering an identity map in $B$ are isomorphisms).

(b) (Source-lifting) For each object $y$ of $\mathscr{C}$ and each morphism $\alpha: a \rightarrow \phi(y)$ in $\mathscr{B}$, there exists a morphism $\beta: x \rightarrow y$ in $\mathscr{C}$ such that $\phi(\beta)=\alpha$.

(c) (Divisibility) For each pair of morphisms $\alpha: x \rightarrow y$ and $\alpha^{\prime}: x^{\prime} \rightarrow$ $y$ in $\mathscr{C}$ and each morphism $\beta: \phi(x) \rightarrow \phi\left(x^{\prime}\right)$ such that $\phi(\alpha)=$ $\phi\left(\alpha^{\prime}\right) \beta$, there exists a morphism $\gamma: x \rightarrow x^{\prime}$ in $\mathscr{C}$ such that $\alpha=\alpha^{\prime} \gamma$ and $\phi(\gamma)=\beta$.

A groupoid $\mathscr{C}$ has unique divisibility if the map $\gamma$ asserted to exist in (c) is unique. All the groupoids we consider will have this property. Notice in particular that $\pi(X ; G)$ is a groupoid over $\mathscr{G}$ with unique divisibility, and that the fiber $\phi^{-1}(G / H)$ is the usual fundamental groupoid $\pi\left(X^{H}\right)$.

Definition 3.2. If $\phi: \mathscr{C} \rightarrow \mathscr{B}$ is a continuous functor of topological categories and $\alpha$ and $b$ are objects in $\mathscr{C}$, let $h \mathscr{C}(a, b)$ be the space obtained from the map $\phi: \mathscr{C}(a, b) \rightarrow \mathscr{B}(\phi(a), \phi(b))$ by replacing each preimage $\phi^{-1}(f)$ with $\pi_{0} \phi^{-1}(f)$, its space of components (using the quotient topology). This gives a new category $h \mathscr{C}$ having the same objects as $\mathscr{C}$, and a functor $h \phi: h \mathscr{C} \rightarrow \mathscr{B}$. Say that $\mathscr{C}$ is a groupoid-like category over $\mathscr{B}$ if $h \phi: h \mathscr{C} \rightarrow \mathscr{B}$ is a groupoid over $\mathscr{B}$. 
Let $h G \mathscr{B}$ be the category of orthogonal $G$-bundles over $G$-orbits and $G$-homotopy classes of linear maps, so there is again a functor $\phi: h G \mathscr{B} \rightarrow \mathscr{G}$, giving the base-space. A representation of $\pi(X ; G)$ is a functor $\rho: \pi(X ; G) \rightarrow h G \mathscr{B}$ such that $\phi \rho=\phi$; that is, it is a functor over $\mathscr{G}$. We add representations in the obvious way, by taking direct sums of bundles. Taking isomorphism classes of representations gives a monoid; applying the Grothendieck construction gives $R O(\pi(X ; G))$, the group of virtual representations of the fundamental groupoid of $X$. If $V$ is a representation of $G$, we will write $V$ also for the representation of $\pi(X ; G)$ that sends every object over $G / H$ to the bundle $G / H \times V$, and every morphism over $\sigma: G / H \rightarrow G / K$ to $\sigma \times 1$. In this way we can think of $R O(G) \subset R O(\pi(X ; G))$.

If $\xi$ is a $G$-bundle over $X$, then $\xi$ determines a representation $\rho(\xi)$ of $\pi(X ; G)$ given by $\rho(\xi)(x: G / H \rightarrow X)=x^{*}(\xi)$ on objects. $\rho(\xi)$ is defined on maps using the lifting property for $G$-bundles. Since $\rho$ preserves addition, it extends to a homomorphism $\rho$ : $K O_{G}(X) \rightarrow R O(\pi(X ; G))$. Let $D O_{G}(X)$ denote the image of $\rho$. Roughly speaking, $D O_{G}(X)$ is the group of equivalence classes of virtual bundles over $X$ where two virtual $G$-bundles are equivalent if they have the same local representations and the same action of the fundamental groupoid on fibers. We think of $\rho(\alpha)$ as the dimension of $\alpha$. The main theorem of this section is:

THEOREM 3.3. If $\alpha$ and $\beta \in K O_{G}(X)$ and $\rho(\alpha)=\rho(\beta)$, then $H_{G}^{\alpha}(Y \rightarrow X ; T) \cong H_{G}^{\beta}(Y \rightarrow X ; T)$.

An immediate consequence of Theorem 3.3 is that we can grade ordinary cohomology on $\mathrm{DO}_{G}(-)$ instead of $K O_{G}(-)$. In fact, the ultimate result in this direction should be that we can grade ordinary cohomology on the group of stable spherical representations of $\pi(X ; G)$; this we may show in a future paper. Another consequence of the theorem, together with Proposition 2.3, is the following.

THEOREM 3.4 (Thom Isomorphism). If $\eta$ is any G-vector bundle over $X$ and if $\alpha \in D O_{G}(X)$, then there is a natural isomorphism

$$
H_{G}^{\alpha}(X ; T) \cong \widetilde{H}_{G}^{\alpha+g \rho(\eta)}(T \eta ; T)
$$

for any coefficient system $T$. In particular, if $\rho(\eta)=V$, then

$$
H_{G}^{\alpha}(X ; T) \cong \widetilde{H}_{G}^{\alpha+V}(T \eta ; T) .
$$


As in Proposition 2.3, we define $\widetilde{H}_{G}^{\alpha+\rho(\eta)}(T \eta ; T)$ to be $H_{G}^{\alpha+\rho(\eta)}(D(\eta), S(\eta) ; T)$ if $\alpha$ and $\eta$ do not extend to the Thom space. The second conclusion of Theorem 3.4 has been previously noticed for $R O(G)$-graded cohomology; the condition $\rho(\eta)=V$ implies that $\eta$ is an "orientable $V$-bundle" in the sense of [W2], but is slightly stronger.

The proof of Theorem 3.3 requires several preliminary results on classifying spaces of groupoids over $\mathscr{G}$. Several such constructions are known; see for example [CMW] for one case. If the groupoids in question have discrete topologies, then there are simpler constructions than the one that follows [M2], but we have need of the topological case. We shall work with a set $G \mathscr{U}_{n}$ of $n$-dimensional $G$-vector bundles over orbits. These $G$-bundles have the form $v(H, V): G \times_{H} V \rightarrow G / H$, where $H \subset G$ and $V \subset \mathscr{U}$ is an $H$-invariant $n$-dimensional subspace. A category over $G \mathscr{U}_{n}$ is a category whose underlying set of objects is $G \mathscr{U}_{n}$ and which comes equipped with a functor $\phi: C \rightarrow \mathscr{G}$ taking the object $v(H, V)$ to $G / H$. If $\mathscr{C}$ is a topological category over $G \mathscr{U}_{n}$, we define the classifying $G$-space of $\mathscr{C}$ by the formula

$$
B_{G} \mathscr{C}=B(*, \mathscr{C}, \phi) \text {. }
$$

Here, $B(-,-,-)$ denotes the two-sided geometric bar construction [M1]. On the simplicial level $B_{k}(*, \mathscr{C}, \phi)$, for $k>0$, consists of tuples $\left(*\left[f_{1}, \ldots, f_{k}\right] g H_{k}\right)$ where $f_{i}: v\left(H_{i}, V_{i}\right) \rightarrow v\left(H_{i-1}, V_{i-1}\right)$ is a morphism in $\mathscr{C}$ and $g H_{k} \in G / H_{k}$. We take $B_{0}(*, \mathscr{C}, \phi)$ to be the space of cosets $\left(*[]_{g} H_{0}\right)_{v}$ indexed on the objects $v=v\left(H_{0}, V_{0}\right)$ in $\mathscr{C}$. $G$ acts on $B_{G} \mathscr{C}$ through its action on the last coordinate.

Following are the examples we shall need.

EXAMPLES 3.5. (i) Take $\mathscr{C}=O_{n}$, the category of pairs $(H, V)$, where $V$ is an $H$-invariant $n$-dimensional subspace of $\mathscr{U}$, and where a morphism $(H, V) \rightarrow(J, W)$ is an orthogonal $G$-bundle map $G \times_{H}$ $V \rightarrow G \times{ }_{J} W$. The resulting space $B_{G} \mathscr{C}$ is the classifying space $B_{G} O_{n}$ for $n$-dimensional orthogonal $G$-bundles [W1], and is $G$-homotopy equivalent to the Grassmannian of $n$-planes in $\mathscr{U}$.

(ii) Take $\mathscr{C}=h O_{n}$, the homotopy category over $\mathscr{G}$ associated with $O_{n}$ by Definition 3.2. If [ $\left.\mathscr{D}, h O_{n}\right]_{\mathscr{G}}$ denotes the set of natural isomorphism classes of maps over $\mathscr{G}$, then $\left[\pi(X ; G), h O_{n}\right]_{\mathscr{G}}$ is the set of isomorphism classes of $n$-dimensional representations of $\pi(X ; G)$. Note that there is a discretization map $B_{G} O_{n} \rightarrow B_{G} h O_{n}$. In Theorem 3.8 we will show that $B_{G} h O_{n}$ classifies representations of the fundamental groupoids of $G$-spaces, so that the discretization map represents the passage from a $G$-vector bundle to the associated groupoid representation. 
(iii) If $V \subset \mathscr{U}$ is an $H$-invariant $n$-dimensional subspace of $\mathscr{U}$, let $K(T \mid H, V)$ be an $H$-equivariant Eilenberg-Mac Lane space of type $(T \mid H, V)$ [LMM, CW]. Take $\mathscr{C}=\mathscr{K} T_{n}$, the category whose morphisms $v(H, V) \rightarrow v(J, W)$ covering $f: G / H \rightarrow G / J$ consist of the fiberwise $G$-homotopy equivalences $G \times{ }_{H} K(T \mid H, V) \rightarrow$ $G \times{ }_{J} K(T \mid J, W)$ covering $f$. The resulting space $B_{G} \mathscr{K} T_{n}$ classifies $G$-fibrations whose fibers over $H$-fixed points are $H$-equivariantly equivalent to $K(T \mid H, V)$ for some $V$, by [W1; 2.3.6]. In particular, in the spectrum $H T$, the fibration $H T_{n} \rightarrow B_{G} O_{n}$ is classified by a $G$-map $\chi: B_{G} O_{n} \rightarrow B_{G} \mathscr{H} T_{n}$.

(iv) Take $\mathscr{C}=h \mathscr{K} T_{n}$, the homotopy category over $\mathscr{G}$ associated with $\mathscr{K} T_{n}$. The morphism spaces $\mathscr{K} T_{n}(a, b)$ have weakly contractible components. To see this, it suffices to show that

$$
\pi_{n} \operatorname{map}(K(T, V), K(T, V))^{K}=0 \quad \text { for } n>0,
$$

but one has that

$$
\pi_{n} \operatorname{map}(K(T, V), K(T, V))^{K} \cong \widetilde{H}_{K}^{V-n}(K(T, V) ; T)=0
$$

for $n>0$. This in turn is true since $\pi_{m}\left(K(T, V)^{J}\right) \cong \widetilde{H}_{J}^{V}\left(S^{m} ; T\right) \cong$ $\widetilde{H}_{m}^{J}\left(S^{V} ; T\right)=0$ for $m<\left|V^{J}\right|$, which, by [L2, 7.1] is enough to show the vanishing of $H_{K}^{V-n}(K(T, V) ; T)$ for $n>0$. It follows that the discretization functor $h: \mathscr{K} T_{n} \rightarrow h \mathscr{K} T_{n}$ is a weak equivalence on morphism spaces. We shall see below that $h$ induces a weak $G$ equivalence of classifying spaces.

If $\alpha$ is an object of $\mathscr{C}$, let $\mathscr{C}_{\alpha}$ be the category of maps out of $\alpha$, or the category of objects under $\alpha$; i.e., the objects are maps $\alpha \rightarrow \beta$ in $\mathscr{C}$ and morphisms are commutative triangles. Also define $\mathscr{C} / \phi$ to be the category whose objects are those of $\mathscr{C}$ and whose morphisms are equivalence classes of morphisms of $\mathscr{C}$ under the identification $f \approx g$ if $\phi(f)=\phi(g)$.

LEMMA 3.6. Let $\mathscr{C}$ be a groupoid-like category over $\mathscr{G}$. Then the space $\left(B_{G} \mathscr{C}\right)^{H}$ is a disjoint union of copies of the connected spaces $B\left(*, \mathscr{C},(\mathscr{C} / \phi)_{\alpha}\right)$, one for each isomorphism class $[\alpha]$ of objects in $h \mathscr{C}$ with $\phi(\alpha)=G / H$.

Proof. Let $\alpha$ be an object in $\mathscr{C}$ with $\phi(\alpha)=G / H$. It is clear that $B\left(*, \mathscr{C},(\mathscr{C} / \phi)_{\alpha}\right) \subset\left(B_{G} \mathscr{C}\right)^{H}$. It follows from the source-lifting property that $\left(B_{G} \mathscr{C}\right)^{H}$ is the union of subspaces of this form. Inclusion of the first simplicial filtration is a $\pi_{0}$-equivalence, and we can use this, together with the divisibility property, to see that if $\alpha$ and $\beta$ 
are non-isomorphic objects of $h \mathscr{C}$ mapping to $G / H$ under $\phi$, then $B\left(*, \mathscr{C},(\mathscr{C} / \phi)_{\alpha}\right)$ and $B\left(*, \mathscr{C},(\mathscr{C} / \phi)_{\beta}\right)$ lie in distinct components of $\left(B_{G} \mathscr{C}\right)^{H}$. Further, each subspace $B\left(*, \mathscr{C},(\mathscr{C} / \phi)_{\alpha}\right)$ may be seen to be connected by again considering $\pi_{0}$. The result now follows by combining this collection of observations.

LeMma 3.7. The map $B_{G} h: B_{G} \mathscr{K} T_{n} \rightarrow B_{G} h \mathscr{K} T_{n}$ is a weak $G$ equivalence.

Proof. Let $H \subset G$; we show that $\left(B_{G} h\right)^{H}$ is a (nonequivariant) weak equivalence. In view of the previous lemma, it suffices to show that, for each $\alpha \in G \mathscr{U}_{n}$, the map

$$
B\left(*, \mathscr{K} T_{n},\left(\mathscr{K} T_{n} / \phi\right)_{\alpha}\right) \rightarrow B\left(*, h \mathscr{K} T_{n},\left(h \mathscr{K} T_{n} / \phi\right)_{\alpha}\right)
$$

is a weak equivalence. For this, consider the diagram

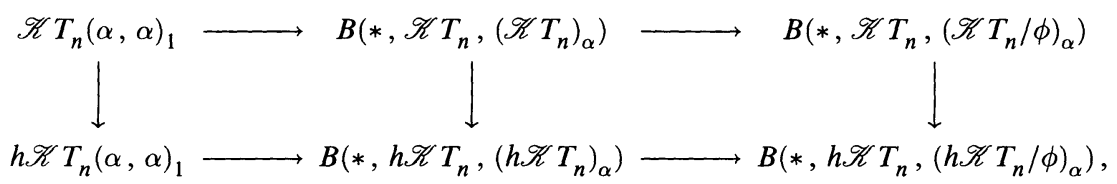

where $\mathscr{C}(\alpha, \alpha)_{1}$ is the space of morphisms $\alpha \rightarrow \alpha$ in $\mathscr{C}$ over the identity on $\phi(\alpha)$. Each row is a quasifibration, using the Dold-Thom criterion; for details, see [M1, §7.6] (our groupoid-like property replaces his group-like assumption). As mentioned in 3.5(iv), the vertical map on the left is a weak equivalence. The spaces in the middle of each line are contractible, so the middle map is also an equivalence. Therefore, the map on the right is a weak equivalence.

Finally, we need the following important result.

THEOREM 3.8. Let $\phi: \mathscr{C} \rightarrow \mathscr{G}$ be a groupoid with unique divisibility such that the topology on $\mathscr{C}$ is discrete. If $X$ has the G-homotopy type of a $G-C W$ complex, then

$$
\left[X, B_{G} \mathscr{C}\right]_{G} \cong[\pi(X ; G), \mathscr{C}]_{\mathscr{G}},
$$

where $[-,-]_{G}$ denotes $G$-homotopy classes of $G$-maps, and $[-,-]_{\mathscr{G}}$ denotes natural isomorphism classes of maps over $\mathscr{G}$. In other words, $B_{G}$ is right adjoint to $\pi$.

Proof. We construct maps in both directions. Start with the map

$$
\Phi:\left[X, B_{G} \mathscr{C}\right]_{G} \rightarrow[\pi(X ; G), \mathscr{C}]_{\mathscr{G}} .
$$


This will be induced by an equivalence of groupoids $\mu: \mathscr{C} \rightarrow$ $\pi\left(B_{G} \mathscr{C} ; G\right)$ over $\mathscr{G}$ (i.e., this will be the inverse of the counit of the adjunction we are constructing). On objects, $\mu$ is specified by taking $\alpha$ to the vertex $\left((*[] e H)_{\alpha} ; 1\right)$ in $\left(B_{G} \mathscr{C}\right)^{H}$, where $\phi(\alpha)=G / H$. (Here we are following May's notation from [M1, §11].) If $\omega: \alpha \rightarrow \beta$ is a morphism in $\mathscr{C}$, then define $\mu(\omega)$ to be the homotopy class of the path $t \mapsto(*[\omega] e H ;(t, 1-t))$. This is a path from $\mu(\alpha)$ to $g \mu(\beta)$, where $\phi(\omega)(e H)=g K$, and thus specifies a morphism in $\pi\left(B_{G} \mathscr{C} ; G\right)$ from $\mu(\alpha)$ to $\mu(\beta)$ covering $\phi(\omega)$. Next, we show that $\mu$ is an equivalence by showing that it restricts to an isomorphism of sketeta. (A skeleton of a groupoid over $\mathscr{G}$ has only one object from each isomorphism class over the identity on each orbit in $\mathscr{G}$.) By Lemma 3.6, the image of a skeleton of $\mathscr{C}$ is a skeleton of $\pi\left(B_{G} \mathscr{C} ; G\right)$, and $\mu$ is a one-to-one correspondence on objects. It remains to show that $\mu$ is bijective on morphisms, and we do this in two steps. First, we show that $\mu$ is bijective on automorphisms over the identity. One has the principal fibration $B\left(*, \mathscr{C}, \mathscr{C}_{\alpha}\right) \rightarrow B\left(*, \mathscr{C},(\mathscr{C} / \phi)_{\alpha}\right)$ with fiber $\operatorname{Aut}(\alpha)$ and contractible total space, where $\operatorname{Aut}(\alpha)$ is the group of automorphisms over $\mathrm{id}_{\phi(\alpha)}$. Consider the commutative diagram

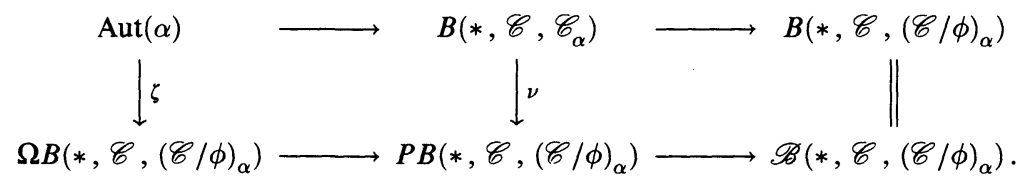

Here the bottom row is the path-space fibration,

$$
\nu\left(*\left[f_{1}, \ldots, f_{n}\right] f ; x\right)(t)=\left(*\left[f_{1}, \ldots, f_{n}, f\right] 1_{\alpha} ;(x t, 1-t)\right),
$$

$\zeta(f)=\left(*[f] 1_{\alpha} ;(t, 1-t)\right)$, and $\mu=\pi_{0}(\zeta)$. Each row is fibration sequence with contractible total space. It now follows that $\zeta$ is a homotopy equivalence on the fibers, and hence that $\mu$ is an isomorphism on maps over the identity. The unique divisibility property now implies that $\mu$ is an isomorphism on sets of morphisms over maps other than the identity. The map $\Phi$ is now specified by $\Phi[f]=\mu^{-1} \circ \pi(f ; G)$.

We now construct the inverse, $\Psi$, of $\Phi$. Start with a map of groupoids $\theta: \pi(X ; G) \rightarrow \mathscr{C} \cong \pi\left(B_{G} \mathscr{C} ; G\right) . \theta$ specifies a well-defined homotopy class of $G$-maps from the 1 -skeleton of $X$ to $B_{G} \mathscr{C}$. Further, we can extend such a map over the 2-skeleton, since $\theta$ is a map of groupoids, so that inessential loops go to inessential loops. Since, by the discussion above, each component $Y$ of each fixed set of $B_{G} \mathscr{C}$ has $\pi_{i}(Y)=0$ if $i \geq 2$, it follows by elementary obstruction theory 
that we can extend the map to all of $X$. We define $\Psi(\theta)$ to be the homotopy class of this extension $\bar{\theta}$.

From the constructions, it is clear that $\Phi \Psi=1$. On the other hand, since it is easy to see that $\bar{\theta}$ is unique up to $G$-homotopy, it follows that $\Phi$ is injective, and we are done.

We can now prove Theorem 3.3.

Proof of Theorem 3.3. If $\alpha$ and $\beta$ are two elements of $K O_{G}(X)$ agreeing in $D O_{G}(X)$, we can assume that $\alpha=[\xi-\eta]$ and $\beta=[\zeta-\eta]$ with $\xi$ and $\zeta$ giving the same representation of $\pi(X ; G)$. Consider the following diagram:

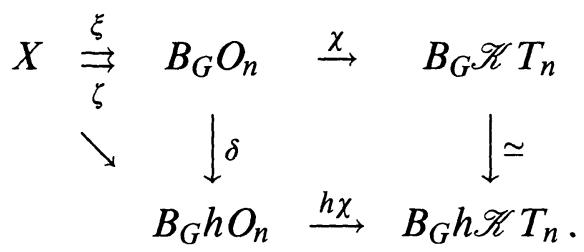

Since $\xi$ and $\zeta$ give the same representation, and since $B_{G} h O_{n}$ classifies representations of $\pi(X ; G)$ by the theorem just proved, the composites $\delta \circ \xi$ and $\delta \circ \zeta$ are $G$-homotopic. It now follows by a diagram chase that $\chi \circ \xi$ and $\chi \circ \zeta$ are $G$-homotopic. Since now the pullbacks along $\xi$ and $\zeta$ of the bundle $H T_{n} \rightarrow B_{G} O_{n}$ are fiber homotopy equivalent, it follows that $H_{G}^{\alpha}(Y \rightarrow X ; T) \cong H_{G}^{\beta}(Y \rightarrow X ; T)$, as claimed.

4. Cohomology of projective spaces. Let $G=\mathbb{Z} / p$. In [L1], Lewis gives an explicit equivariant cell structure for the complex projective space $\mathbb{C} P(V)=S(V) / U(1)$, where $S(V)$ is the unit sphere of the unitary representation $V$. He then uses this decomposition to compute its ordinary $R O(G)$-graded cohomology with Burnside coefficients. Here we show how to obtain the same result using the Thom isomorphism theorem, and also extend the calculation to a larger grading.

Before stating the result, we need some notation. Since $G=\mathbb{Z} / p$, we can decompose $V$ as a sum of one-dimensional unitary representations: $V=L_{0} \oplus L_{1} \oplus \cdots$. Let $\lambda_{0}=0$, and for each $i>0$, let

$$
\lambda_{i}=\sum_{j=0}^{i-1} \bar{L}_{i} \otimes_{\mathbb{C}} L_{j} \text {. }
$$

For $i \geq 0$ let

$$
n_{i}=\operatorname{dim}_{\mathbb{R}} \lambda_{i}^{G}
$$


Thus, $n_{i}$ is twice the number of times $L_{i}$ appears up to isomorphism in $\sum_{j=0}^{i-1} L_{j}$. If $\alpha \in K O_{G}(\mathbb{C} P(V))$, let $\alpha_{i}$ be the restriction of $\alpha$ to the point $\mathbb{C} P\left(L_{i}\right)$, thought of as a representation of $G$, i.e. $\alpha_{i} \in R O(G)$. We shall denote ordinary cohomology with Burnside coefficients by $H_{G}^{*}(-)$.

Theorem 4.1. Assume that $\alpha \in K O_{G}(\mathbb{C P}(V))$ and that

$$
\operatorname{dim}_{\mathbb{R}}\left(\alpha_{i}\right)^{G}-\operatorname{dim}_{\mathbb{R}}\left(\alpha_{j}\right)^{G} \leq n_{i}-n_{j} \text { whenever } i>j .
$$

Then

$$
H_{G}^{\alpha}(\mathbb{C} P(V)) \cong \sum_{i \geq 0} H_{G}^{\alpha_{t}-\lambda_{t}}(*)
$$

Further, if we grade on the coset $\alpha+R O(G)$, then this isomorphism is one of modules over the $R O(G)$-graded cohomology of a point.

The following is now an immediate consequence of the theorem.

Corollary 4.2 (L. G. Lewis). If $\left(n_{i}\right)$ is a non-decreasing sequence, then the conclusion of Theorem 4.1 holds for any $\alpha \in R O(G)$.

Lewis also computes the multiplicative structure, and this is far more complicated. For details, see [L1]. Both our result and Lewis' hinge on explicit information about the $R O(G)$-graded cohomology of a point as first calculated by Stong (unpublished) and we state what little we shall need in the following lemma. (A complete description of the cohomology of a point appears in [L1].)

Lemma 4.3. Let $G=\mathbb{Z} / p$ and $\sigma \in R O(G)$. Then $H_{G}^{\sigma}(*)=0$ when $\operatorname{dim}_{\mathbb{R}}(\sigma)$ and $\operatorname{dim}_{\mathbb{R}}\left(\sigma^{G}\right)$ are odd, $\operatorname{dim}_{\mathbb{R}}(\sigma)<0$, and $\operatorname{dim}_{\mathbb{R}}\left(\sigma^{G}\right) \leq 1 . \square$

Proof of Theorem 4.1. We first assume that $V$ is finite dimensional, and induct on $m=\operatorname{dim}_{\mathbb{C}} V$. The beginning of the induction, $m=1$, is trivial. For the inductive step, we consider the inclusion

$$
l: \mathbb{C} P\left(L_{1} \oplus \cdots \oplus L_{n}\right) \hookrightarrow \mathbb{C} P\left(L_{0} \oplus L_{1} \oplus \cdots \oplus L_{n}\right) .
$$

By a standard geometric argument, the normal bundle of $l$ is $\nu=$ $\operatorname{Hom}\left(\xi, L_{0}\right)=\bar{\xi} \otimes L_{0}$, where $\xi$ is the canonical line bundle over the subspace $\mathbb{C} P\left(L_{1} \oplus \cdots \oplus L_{n}\right)$, and the associated Thom space is $\mathbb{C} P\left(L_{0} \oplus L_{1} \oplus \cdots \oplus L_{n}\right)$ with basepoint $\mathbb{C} P\left(L_{0}\right)$. By the Thom isomorphism,

$$
\widetilde{H}_{G}^{\alpha}\left(\mathbb{C} P\left(L_{0} \oplus L_{1} \oplus \cdots \oplus L_{n}\right)\right) \cong H_{G}^{\alpha-\nu}\left(\mathbb{C} P\left(L_{1} \oplus \cdots \oplus L_{n}\right)\right) .
$$


(Note that $\alpha$ and $\nu$ are defined on all of $\mathbb{C} P\left(L_{0} \oplus L_{1} \oplus \cdots \oplus L_{n}\right)$.) By induction, $H_{G}^{\alpha-\nu}\left(\mathbb{C} P\left(L_{1} \oplus \cdots \oplus L_{n}\right)\right) \cong \sum_{i>0} H_{G}^{\alpha_{i}-\lambda_{i}}(*)$, where $\lambda_{i}=$ $\sum_{0 \leq j \leq i-1} L_{i} \otimes_{\mathbb{C}} L_{j}$ and where the hypothesis on $\alpha-\nu$ and the sequence $L_{1}, \ldots, L_{n}$ may be checked to hold. The long exact sequence of the pair $\left(\mathbb{C} P(V), \mathbb{C} P\left(L_{0}\right)\right.$ now becomes

$$
\cdots \rightarrow \sum_{i>0} H_{G}^{\alpha_{i}-\lambda_{l}}(*) \rightarrow H_{G}^{\alpha}(\mathbb{C} P(V)) \rightarrow H_{G}^{\alpha_{0}}(*) \stackrel{\partial}{\longrightarrow} \sum_{i>0} H_{G}^{\alpha_{t}-\lambda_{l}+1}(*) \rightarrow \cdots
$$

We claim that the connecting homomorphism $\partial$ is zero. To see this, it suffices to show that $\partial$ is zero with $\alpha$ replaced by $\alpha-\alpha_{0}$, since $\partial$ is a map of $R O(G)$-graded $H_{G}^{*}(*)$-modules and the generator of $H_{G}^{*}(*)$ lies in degree 0 . However, the summands $H_{G}^{\alpha_{t}-\alpha_{0}-\lambda_{t}+1}(*)$ are all zero, by Lemma 4.3. Since $H_{G}^{*}(*)$ is projective as a module over itself, the resulting short exact sequence splits, completing the inductive step, and hence the proof for the case $V$ finite.

If $V$ is countably infinite dimensional, the result follows by passage to inverse limits-the $\lim ^{1}$-terms vanishing since the maps of the system are epimorphisms.

Appendix. Virtual bundles. We construct, carefully, a category of virtual bundles, in order to explain how to reduce the grading of cohomology theories given in $\S 2$ from pairs of bundles to a category whose isomorphism classes give $K O_{G}(X)$, and obtain a well-defined functor of $X$. This construction is essentially the one that appears in [CMW].

Definition A1. (a) Let $\mathscr{U}$ be a $G$-universe. Define $G \mathscr{V}$ to be the category whose objects are pairs $(X, \xi)$, where $X$ is a $G$-space and $\xi$ is a finite-dimensional $G$-subbundle of $X \times \mathscr{U}$. The morphisms are the $G$-bundle maps. We shall usually write $\xi$ for $(X, \xi)$.

(b) Let $\mathscr{S} G \mathscr{V}$ be the category whose objects are pairs of bundles over the same base space, and whose maps $(\xi, \zeta) \rightarrow\left(\xi^{\prime}, \zeta^{\prime}\right)$ are equivalence classes of pairs of $G$-bundle maps $(f, g):(\xi+\eta, \zeta+\eta) \rightarrow$ $\left(\xi^{\prime}+\eta^{\prime}, \zeta^{\prime}+\eta^{\prime}\right)$ covering the same map of base spaces. The equivalence relation is the one generated by three elementary equivalences. First, $(f, g) \approx\left(f+\mathrm{id}_{\mu}, g+\mathrm{id}_{\mu}\right)$, where, if $\xi^{\prime}$ and $\zeta^{\prime}$ are bundles over $X$ and $f$ and $g$ cover the map $F$ on base spaces, then $\mu$ is any subbundle of $X \times \mathscr{U}$ perpendicular to $\xi^{\prime}+\eta^{\prime}$ and $\zeta^{\prime}+\eta^{\prime}$, with $F^{*} \mu$ perpendicular to $\xi+\eta$ and $\zeta+\eta$, and by $\operatorname{id}_{\mu}$ we mean the canonical map $F^{*} \mu \rightarrow \mu$. 
Secondly, let

$$
\begin{aligned}
& (f, g):(\xi+\eta, \zeta+\eta) \rightarrow\left(\xi^{\prime}+\eta^{\prime}, \zeta^{\prime}+\eta^{\prime}\right) \\
& \quad \approx(h, k):(\xi+\mu, \zeta+\mu) \rightarrow\left(\xi^{\prime}+\mu^{\prime}, \zeta^{\prime}+\mu^{\prime}\right)
\end{aligned}
$$

if there are bundle maps $\eta \rightarrow \mu$ and $\eta^{\prime} \rightarrow \mu^{\prime}$ making the following diagrams both fiber homotopy commute:

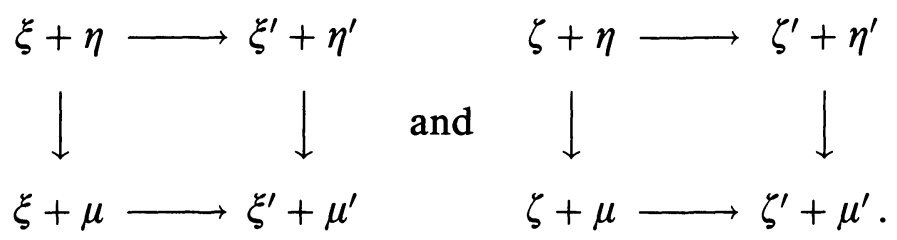

The last relation is fiber-homotopy of maps (over a fixed base map). We call the objects of $\mathscr{S} G \mathscr{V}$ virtual bundles, and the morphisms virtual bundle maps.

(c) If $X$ is a $G$-space, let $\mathscr{S} G \mathscr{V}(X)$ be the subcategory of $\mathscr{S} G \mathscr{V}$ consisting of virtual bundles over $X$ and maps over the identity map of $X$.

We are interested mainly in the isomorphism classes of these categories, for we have:

Proposition A.2. There is a natural one-to-one correspondence between the isomorphism classes of objects of $\mathscr{S} G \mathscr{V}(X)$ and the elements of $K O_{G}(X)$.

Proof. The correspondence is given by taking the isomorphism class of the pair $(\xi, \zeta)$ to the element $[\xi-\zeta] \in K O_{G}(X)$, and it is easy to check that this gives a well-defined bijection. In particular, notice that the elementary equivalences of A.1(b) above add no new isomorphisms.

Now let $G E x$ be the category of $G$-ex-spaces. Both $G E x$ and $\mathscr{S} G \mathscr{V}$ map to the category $G \mathscr{U}$ of $G$-spaces by taking base-spaces, and we can form the pullback category $G E x \times_{G \mathscr{U}} \mathscr{S} G \mathscr{V}$, the category of $G$-ex-spaces and virtual bundles on the base-spaces.

The following result shows the sense in which we can grade cohomology theories of $K O_{G}(X)$. It also shows in what sense it is homotopy invariant.

THeOREM A3. (i) If $E$ is a G-spectrum and $(W \rightarrow X, \xi, \zeta)$ is an object of $G E x \times_{G \mathscr{U}} h \mathscr{S} G \mathscr{V}$, then the assignment $(W \rightarrow X, \xi, \zeta) \mapsto$ $\widetilde{E}_{G}^{\xi-\zeta}(W \rightarrow X)$ defines a contravariant functor on $G E x \times_{G \mathscr{U}} h \mathscr{S} G \mathscr{V}$. 
(ii) The functor of part (i) factors through the homotopy category of $G E x \times_{G \mathscr{U}} h \mathscr{S} G \mathscr{V}$.

Proof. (i) Recall that an element of $\widetilde{E}_{G}^{\xi^{\prime}-\zeta^{\prime}}(W \rightarrow X)$ is a homotopy class of maps making the diagram

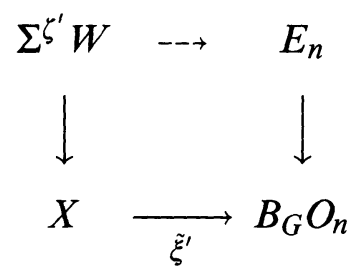

commute, where $\tilde{\xi}^{\prime}$ is the Gauss map. Now suppose given a $G$-ex-map $(\widetilde{F}, F):(Y \rightarrow Z) \rightarrow(W \rightarrow X)$, with $\widetilde{F}: Y \rightarrow W$ and $F: Z \rightarrow X$. Suppose also given a virtual bundle map $(\xi, \zeta) \rightarrow\left(\xi^{\prime}, \zeta^{\prime}\right)$ over $F$, represented by the pair of bundle maps $(f, g):(\xi+\eta, \zeta+\eta) \rightarrow$ $\left(\xi^{\prime}+\eta^{\prime}, \zeta^{\prime}+\eta^{\prime}\right)$. Starting with $\alpha \in \widetilde{E}_{G}^{\xi^{\prime}-\zeta^{\prime}}(W \rightarrow X)$ represented by the diagram above, we suspend the diagram by $\eta^{\prime}$, obtaining

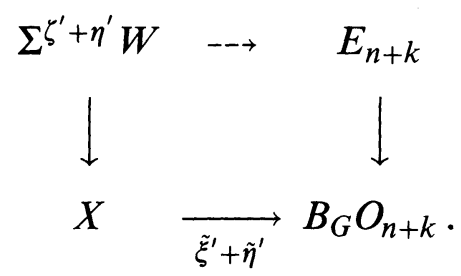

Now, the bundle map $\xi+\eta \rightarrow \xi^{\prime}+\eta^{\prime}$ specifies a homotopy class of homotopies from $\tilde{\xi}+\tilde{\eta}$ to $\left(\tilde{\xi}^{\prime}+\tilde{\eta}^{\prime}\right) \circ F$; using the covering homotopy property of fiber bundles, and the $G$-map $\Sigma^{\zeta+\eta} Z \rightarrow \Sigma^{\zeta^{\prime}+\eta^{\prime}} W$ specified by $\widetilde{F}$ and the bundle map $\zeta+\eta \rightarrow \zeta^{\prime}+\eta^{\prime}$, gives us a commutative diagram

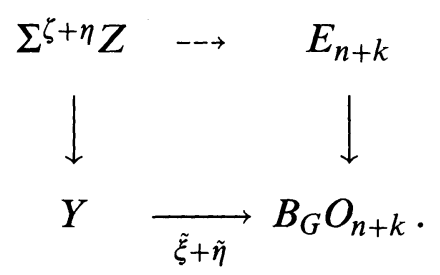

Finally, we can take adjoints to obtain the diagram

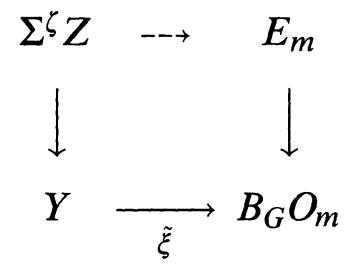


representing an element of $\widetilde{E}_{G}^{\xi-\zeta}(Y \rightarrow X)$ (for details about the suspension and adjunction used, see [E]). This defines the map

$$
\widetilde{E}_{G}^{\xi^{\prime}-\zeta^{\prime}}(W \rightarrow X) \rightarrow E_{G}^{\xi-\zeta}(Z \rightarrow Y) .
$$

We can write what we just did as defining a sequence of homomorphisms

$$
\begin{aligned}
\widetilde{E}_{G}^{\xi^{\prime}-\zeta^{\prime}}(W \rightarrow X) & \rightarrow \widetilde{E}_{G}^{\left(\xi^{\prime}+\eta^{\prime}\right)-\left(\zeta^{\prime}+\eta^{\prime}\right)}(W \rightarrow X) \\
& \rightarrow \widetilde{E}_{G}^{(\xi+\eta)-(\zeta+\eta)}(Z \rightarrow Y) \rightarrow \widetilde{E}_{G}^{\xi-\zeta}(Z \rightarrow Y) .
\end{aligned}
$$

We now need to show that this construction respects the elementary equivalences given in A.1.(b) above. That it respects the first is easy to see. For the second, suppose given maps $\eta \rightarrow \mu$ and $\eta^{\prime} \rightarrow \mu^{\prime}$ making the diagram in A.1.(b) commute, we can form the following commutative diagram:

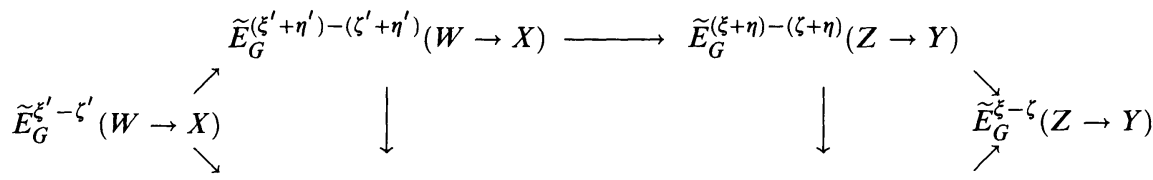

$$
\begin{aligned}
& \widetilde{E}_{G}^{\left(\xi^{\prime}+\mu^{\prime}\right)-\left(\zeta^{\prime}+\mu^{\prime}\right)}(W \rightarrow X) \longrightarrow \widetilde{E}_{G}^{(\xi+\mu)-(\zeta+\mu)}(Z \rightarrow Y) .
\end{aligned}
$$

The vertical arrow are defined in the same way that the middle arrow was defined in the step above. One can now check that the outer triangles and middle square of this diagram commute, showing that the paths around the top and the bottom give the same homomorphism.

Finally, to see that this construction respects fiber-homotopy is straightforward, since we are only concerned with homotopy classes in the defining diagram.

For (ii), again we are only concerned with homotopy classes, so this is straightforward.

One should not expect to make cohomology into a functor on the obvious category of pairs $\left(Z \rightarrow X, \alpha \in K O_{G}(X)\right)$, as the virtual bundle maps are crucial to the functoriality and there is no canonical way of choosing such maps in this situation.

\section{REFERENCES}

[CMW] S. R. Costenoble, J. P. May and S. Waner, Equivariant orientation theory, preprint, 1989.

[CW] S. R. Costenoble and S. Waner, Fixed set systems of equivariant loop spaces, Trans. Amer. Math. Soc., 326 (1991), 485-505. 
[C] M. C. Crabb, $\mathbb{Z} / 2$-homotopy theory, London Math. Soc. Lecture Notes, 44 (1980).

[E] A. D. Elmendorf, The Grassmannian geometry of spectra, J. Pure \& Appl. Algebra, 54 (1988), 37-94.

[J] I. M. James, Ex-homotopy theory I, Illinois J. Math., 15 (1971), 324-337.

[L1] L. G. Lewis Jr., The $R O(G)$-graded equivariant ordinary cohomology of complex projective spaces with linear $\mathbb{Z} / p$ actions, Lecture Notes in Math., 1361 Springer-Verlag (1988), 53-122.

[L2] L. G. Lewis Jr., The equivariant Hurewicz map, preprint.

[LMM] G. L. Lewis Jr., J. P. May and J. McClure, Ordinary RO $(G)$-graded equivariant cohomology, Bull. Amer. Math. Soc., 4 (1981), 208-212.

[LMS] L. G. Lewis Jr., J. P. May and M. Steinberger, with contributions by J. McClure, Equivariant stable homotopy theory, Lecture Notes in Math., 1213, Springer-Verlag (1986).

[M1] J. P. May, Classifying spaces and fibrations, Mem. Amer. Math. Soc., 155 (1975).

[M2] J. P. May, G-spaces and fundamental groupoids, preprint.

[W1] S. Waner, Equivariant classifying spaces and fibrations, Trans. Amer. Math. Soc., 258 (2) (1980), 385-405.

[W2] S. Waner, Classification of oriented equivariant spherical fibrations, Trans. Amer. Math. Soc., 271 (1) (1982), 313-324.

Received May 9, 1990 and in revised form January 25, 1991.

HOFSTRA UNIVERSITY

HEMPSTEAD, NY 11550 



\title{
PACIFIC JOURNAL OF MATHEMATICS EDITORS
}

\author{
V. S. VARADARAJAN \\ (Managing Editor) \\ University of California \\ Los Angeles, CA 90024-1555-05 \\ Herbert Clemens \\ University of Utah \\ Salt Lake City, UT 84112 \\ F. Michael Christ \\ University of California \\ Los Angeles, CA 90024-1555 \\ THOMAS ENRIGHT \\ University of California, San Diego \\ La Jolla, CA 92093
}

Nicholas ERcolani

University of Arizona

Tucson, AZ 85721

R. FINN

Stanford University

Stanford, CA 94305

VAUGHAN F. R. JoNeS

University of California

Berkeley, CA 94720

C. C. MOORE

University of California

Berkeley, CA 94720

MARTIN SchaRLEMANN

University of California

Santa Barbara, CA 93106

HAROLd StaRK

University of California, San Diego

La Jolla, CA 92093

SteVen Kerckhoff

Stanford University

Stanford, CA 94305

\section{ASSOCIATE EDITORS}

\begin{tabular}{|c|c|c|c|c|}
\hline R. ARENS & $\begin{array}{l}\text { E. F. BECKENBACH } \\
(1906-1982)\end{array}$ & NeumanN & $\begin{array}{c}\text { F. WoLF } \\
(1904-1989)\end{array}$ & K. YoshidA \\
\hline \multicolumn{5}{|c|}{ SUPPORTING INSTITUTIONS } \\
\hline \multicolumn{2}{|c|}{ JNIVERSITY OF ARIZONA } & \multicolumn{3}{|c|}{ UNIVERSITY OF OREGON } \\
\hline \multicolumn{2}{|c|}{ UNIVERSITY OF BRITISH COLUMBIA } & \multicolumn{3}{|c|}{ UNIVERSITY OF SOUTHERN C } \\
\hline \multicolumn{2}{|c|}{ CALIFORNIA INSTITUTE OF TECHNOLOGY } & \multirow{2}{*}{\multicolumn{3}{|c|}{ STANFORD UNIVERSITY }} \\
\hline \multicolumn{2}{|c|}{ UNIVERSITY OF CALIFORNIA } & & & UNIVERSITY OF HAWAII \\
\hline \multicolumn{2}{|c|}{ MONTANA STATE UNIVERSITY } & \multicolumn{3}{|c|}{ UNIVERSITY OF TOKYO } \\
\hline \multicolumn{2}{|c|}{ UNIVERSITY OF NEVADA, RENO } & \multicolumn{3}{|c|}{ UNIVERSITY OF UTAH } \\
\hline \multicolumn{2}{|c|}{ NEW MEXICO STATE UNIVERSITY } & \multicolumn{3}{|c|}{ DN STATE UNIVERSITY } \\
\hline \multicolumn{2}{|c|}{ OREGON STATE UNIVERSITY } & UNIVER & OF WASHIN & \\
\hline
\end{tabular}




\section{Pacific Journal of Mathematics}

Vol. 152, No. $1 \quad$ January, 1992

B. V. Rajarama Bhat, On a characterization of velocity maps in the space of observables .......................................

John David Brillhart, Note on the discriminant of certain cyclotomic period

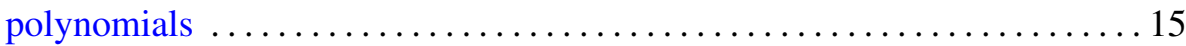

Steven R. Costenoble and Stefan Waner, The equivariant Thom

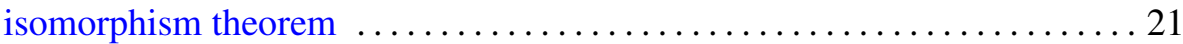

Karl Heinz Dovermann and Dong Youp Suh, Smith equivalence for finite

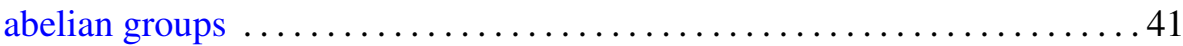

Klaus-Jochen Engel, On singular perturbations of second order Cauchy

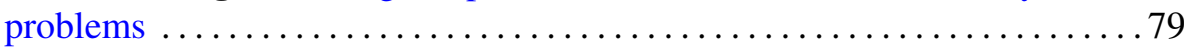

Angel Ferrandez and Pascual Lucas, On surfaces in the 3-dimensional

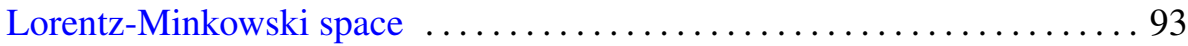

Brian Hartley, A general Brauer-Fowler theorem and centralizers in locally

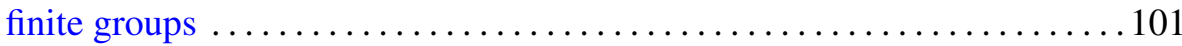

Joel Hass, Intersections of least area surfaces .................... 119

Edward Kissin, Indices of unbounded derivations of $C^{*}$-algebras $\ldots \ldots \ldots 125$

Erhard Luft and Denis Karmen Sjerve, On regular coverings of

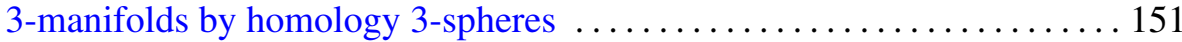

Daniel Pecker, Sur la projection de variétés algébriques réelles .......... 165

Claus Scheiderer, Some remarks on orderings under finite field

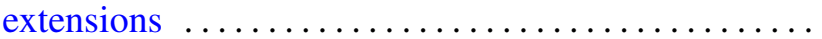

Fernando Serrano, Elliptic surfaces with an ample divisor of genus two 